\title{
POLYNOMIAL RECURRENCES AND CYCLIC RESULTANTS
}

\author{
CHRISTOPHER J. HILLAR AND LIONEL LEVINE
}

(Communicated by Bernd Ulrich)

\begin{abstract}
Let $K$ be an algebraically closed field of characteristic zero and let $f \in K[x]$. The $m$-th cyclic resultant of $f$ is$$
r_{m}=\operatorname{Res}\left(f, x^{m}-1\right) .
$$

A generic monic polynomial is determined by its full sequence of cyclic resultants; however, the known techniques proving this result give no effective computational bounds. We prove that a generic monic polynomial of degree $d$ is determined by its first $2^{d+1}$ cyclic resultants and that a generic monic reciprocal polynomial of even degree $d$ is determined by its first $2 \cdot 3^{d / 2}$ of them. In addition, we show that cyclic resultants satisfy a polynomial recurrence of length $d+1$. This result gives evidence supporting the conjecture of Sturmfels and Zworski that $d+1$ resultants determine $f$. In the process, we establish two general results of independent interest: we show that certain Toeplitz determinants are sufficient to determine whether a sequence is linearly recurrent, and we give conditions under which a linearly recurrent sequence satisfies a polynomial recurrence of shorter length.
\end{abstract}

\section{INTRODUCTION}

Let $K$ be an algebraically closed field of characteristic zero. Given a monic polynomial

$$
f(x)=\prod_{i=1}^{d}\left(x-\lambda_{i}\right) \in K[x],
$$

the $m$-th cyclic resultant of $f$ is

$$
r_{m}(f)=\operatorname{Res}\left(f, x^{m}-1\right)=\prod_{i=1}^{d}\left(\lambda_{i}^{m}-1\right) .
$$

One motivation for the study of cyclic resultants comes from topological dynamics. Sequences of the form (1.1) count periodic points for toral endomorphisms. If $A=\left(a_{i j}\right)$ is a $d \times d$ integer matrix, then $A$ defines an endomorphism $T$ of the $d$-dimensional torus $\mathbb{T}^{d}=\mathbb{R}^{d} / \mathbb{Z}^{d}$ given by

$$
T(\mathbf{x})=A \mathbf{x} \bmod \mathbb{Z}^{d} .
$$

Received by the editors November 23, 2004 and, in revised form, February 8, 2006.

2000 Mathematics Subject Classification. Primary 11B37, 14Q99; Secondary 15A15, 20M25.

Key words and phrases. Cyclic resultants, linear recurrence, polynomial recurrence, semigroup algebra, Toeplitz determinant, topological dynamics, Vandermonde determinant.

Both authors were supported under a NSF Graduate Research Fellowship. 
Let $\operatorname{Per}_{m}(T)=\left\{\mathbf{x} \in \mathbb{T}^{d}: T^{m}(\mathbf{x})=\mathbf{x}\right\}$ be the set of points on the torus fixed by the map $T^{m}$. Under the ergodicity condition that no eigenvalue of $A$ is a root of unity, it follows (see [5]) that

$$
\# \operatorname{Per}_{m}(T)=\left|\operatorname{det}\left(A^{m}-\mathrm{Id}\right)\right|=\left|r_{m}(f)\right|,
$$

in which $f$ is the characteristic polynomial of $A$.

In connection with number theory, cyclic resultants were also studied by Pierce and Lehmer [5] in the hope of using them to produce large primes. As a simple example, the Mersenne numbers $M_{m}=2^{m}-1$ arise as cyclic resultants of the polynomial $f(x)=x-2$. Indeed, the map $T(x)=2 x \bmod 1$ has precisely $M_{m}$ points of period $m$. Further motivation comes from knot theory [14, Lagrangian mechanics [7, 9], and, more recently, the study of amoebas of varieties [13] and quantum computing [10].

The problem of recovering a polynomial from its sequence of cyclic resultants arises naturally in several applications. Commonly, an explicit bound $N=N(d)$ is desired in terms of the degree $d$ of $f$ so that the first $N$ resultants $r_{1}, \ldots, r_{N}$ determine $f$ (see [9, 10]). For instance, given a toral endomorphism of the type discussed above, one would like to use a minimal amount of (coarse) period data to recover the spectrum of the matrix $A$. In general, reconstruction of a polynomial from its sequence of cyclic resultants seems to be a difficult problem. While it is known 8 that in many instances the full sequence of resultants determines $f$, this result is of little use in computation. One purpose of the present article is to give explicit upper bounds on the complexity of this problem. Our main result in this direction is

Theorem 1.1. A generic monic polynomial $f(x) \in K[x]$ of degree $d$ is determined by its first $2^{d+1}$ cyclic resultants $r_{1}, \ldots, r_{2^{d+1}}$. A generic monic reciprocal polynomial of even degree $d$ is determined by its first $2 \cdot 3^{d / 2}$ cyclic resultants.

Emperical evidence suggests that Theorem 1.1 is far from tight. A conjecture of Sturmfels and Zworski addresses the special case of a reciprocal polynomial $f$, that is, one satisfying $f(1 / x)=x^{d} f(x)$.

Conjecture 1.2. A reciprocal monic polynomial $f(x) \in K[x]$ of even degree $d$ is determined by its first $d / 2+1$ cyclic resultants.

Recently, there has been some progress on this conjecture for a special class of reciprocal polynomials. Kedlaya 10 , has shown that for a certain reciprocal polynomial $f$ of degree $d$ arising from the numerator $P(t)$ of a zeta function of a curve over a finite field $\mathbb{F}_{q}$, the first $d$ resultants are sufficient to recover $f$. He uses this result to give a quantum algorithm that computes $P(t)$ in time polynomial in the degree of the curve and $\log q$. A proof of Conjecture 1.2 would further reduce the running time for Kedlaya's algorithm. We offer the following related conjecture.

Conjecture 1.3. A generic monic polynomial $f(x) \in K[x]$ of degree $d$ is determined by its first $d+1$ cyclic resultants.

Presently Conjecture 1.3 is verified only up to $d=4$ (see Section [5); however, we are able to offer a result in the direction of Conjectures 1.2 and 1.3. We say that a sequence $\left\{a_{n}\right\}_{n \geq 1}, a_{n} \in K$, obeys a polynomial recurrence of length $\ell$ if there is a polynomial $P \in K\left[x_{1}, \ldots, x_{\ell}\right]$ such that $P\left(a_{n}, \ldots, a_{n+\ell-1}\right)=0$ for all $n \geq 1$. Our theorem may then be stated as follows. 
Theorem 1.4. Let $f \in K[x]$ be a monic polynomial of degree $d$. The sequence $\left\{r_{n}\right\}_{n \geq 1}$ of cyclic resultants of $f$ obeys a polynomial recurrence of length $d+1$. Moreover if $f$ is assumed reciprocal of even degree $d$, then $\left\{r_{n}\right\}$ obeys a polynomial recurrence of length $d / 2+1$.

Explicit polynomial recurrences witnessing Theorem 1.4 in the cases $d=1,2$ may be found in Section 5 .

The main tools used in our analysis are two general results relating linear and polynomial recurrences. Let $\left\{a_{n}\right\}_{n \geq 1}$ be given by

$$
a_{n}=\sum_{i=1}^{t} p_{i}(n) \mu_{i}^{n}
$$

where the $p_{i}$ are nonzero polynomials in $K[x]$, and the $\mu_{i}$ are nonzero elements of $K$. Let $\ell_{i}=\operatorname{deg}\left(p_{i}\right)+1$. It is well known (see, e.g. [4) that the sequence $a$ obeys a linear recurrence of length $\ell=\ell_{1}+\cdots+\ell_{t}$; namely,

$$
a_{n+\ell}+c_{1} a_{n+\ell-1}+\cdots+c_{\ell} a_{n}=0, \quad n \geq 1,
$$

with coefficients $c_{i}$ determined by

$$
x^{\ell}+c_{1} x^{\ell-1}+\cdots+c_{n}=\prod\left(x-\mu_{i}\right)^{\ell_{i}} .
$$

We say that $a$ obeys a simple linear recurrence if all $\ell_{i}=1$.

The following result gives conditions under which a linearly recurrent sequence satisfies a polynomial recurrence of shorter length.

Theorem 1.5. Let $\left\{a_{n}\right\}_{n \geq 1}$ be given by (1.2), and let $r$ be the rank of the multiplicative group $A \subset K^{*}$ generated by the $\mu_{i}$. Then the sequence $\left\{a_{n}\right\}$ obeys a polynomial recurrence of length $r+2$. Moreover, if $\left\{a_{n}\right\}$ satisfies a simple linear recurrence, then $\left\{a_{n}\right\}$ obeys a polynomial recurrence of length $r+1$.

Example 1.6. Consider the Fibonacci sequence $F_{n}=\frac{1}{\sqrt{5}}\left(\mu_{+}^{n}-\mu_{-}^{n}\right)$, where $\mu_{ \pm}=$ $\frac{1 \pm \sqrt{5}}{2}$. Since $\mu_{-}=-\mu_{+}^{-1}$, the group $A$ in Theorem 1.5 is generated by $\mu_{+}$and -1 , hence it has rank $r=1$. As $F_{n}$ obeys a simple linear recurrence, we expect the sequence $\left\{F_{n}\right\}$ to obey a polynomial recurrence of length $r+1=2$. Indeed, it is well known and easily seen by induction that $F_{n}^{2}-F_{n} F_{n-1}-F_{n-1}^{2}=(-1)^{n}$, so every pair $\left(F_{n-1}, F_{n}\right)$ lies on the zero-locus of $P(x, y)=\left(y^{2}-x y-x^{2}\right)^{2}-1$.

Example 1.7. Let $a_{n}=q(n)$ be a quasi-polynomial of degree $d$ and period $N$; that is, there are polynomials $q_{0}, q_{1}, \ldots, q_{N-1} \in K[x]$ of degree at most $d$, such that $q(n)=q_{i}(n)$ whenever $n \equiv i(\bmod N)$. It is elementary that any such sequence can be expressed in the form (1.2) with $t=d$ and $\mu_{i}=\zeta^{i-1}$, where $\zeta$ is a primitive $d$-th root of unity. Since the group $A \simeq \mathbb{Z} / d \mathbb{Z}$ has rank zero, Theorem 1.5] asserts that the sequence $a_{n}$ obeys a polynomial recurrence of length 2. By contrast, for suitably chosen $q$ the shortest linear recurrence for $a_{n}$ has length $(d+1) N+1$.

We remark that Theorem 1.5 does not always give the shortest length of a polynomial recurrence. For instance, the sequence $a_{n}=2^{n}+(-2)^{n}+3^{n}+(-3)^{n}$ satisfies the length- 2 polynomial recurrence $a_{n} a_{n+1}=0$, while the theorem only guarantees the existence of a recurrence of length $r+1=3$.

The second general result we use gives a polynomial recurrence of length $2 \ell-1$ which "detects" for the existence of a (homogeneous) linear recurrence of length at 
most $\ell$. Given a sequence $\left\{a_{n}\right\}_{n \geq 1}$, consider the $\ell \times \ell$ Toeplitz matrix

$$
A_{\ell, n}=\left[\begin{array}{cccc}
a_{n} & a_{n+1} & \ldots & a_{n+\ell-1} \\
a_{n-1} & a_{n} & \ldots & a_{n+\ell-2} \\
\vdots & \vdots & \ddots & \vdots \\
a_{n-\ell+1} & a_{n-\ell+2} & \ldots & a_{n}
\end{array}\right] .
$$

Theorem 1.8. The sequence $\left\{a_{n}\right\}_{n \geq 1}$ satisfies a homogeneous linear recurrence of length at most $\ell$ if and only if every Toeplitz determinant $\operatorname{det} A_{\ell, n}$ vanishes, $n \geq \ell$.

Although this result appears to be known in some form (for example, it is implicit in the treatment of "number walls" in [1), we include a proof in Section 2 as we were unable to find a reference.

All linear recurrence relations in this paper will henceforth be assumed homogeneous.

Example 1.9. In the case $\ell=2$, the theorem asserts that $a_{n}$ is an exponential sequence $c \mu^{n}$ if and only if $a_{n}^{2}=a_{n-1} a_{n+1}$ for all $n$.

The rest of the paper is organized as follows. In Section 2, we prove Theorems 1.5 and 1.8. The proof the former theorem reduces essentially to the computation of the Krull dimension of a semigroup algebra, and that of the latter theorem to an inductive application of Dodgson's rule. In Section 3, we establish a Toeplitz determinant factorization which will be used in the proof of Theorem 1.1. along with some related factorizations of independent interest. In Section 4 we apply these results, together with those of [8, to prove Theorems 1.1] and 1.4 Finally, in Section 5, we present computational evidence supporting Conjecture 1.3 .

We thank Bernd Sturmfels and Maciej Zworski for bringing this problem to our attention and for useful discussions.

\section{LiNEAR AND POLYNOMIAL RECURRENCES}

Let $S$ denote the collection of all sequences $\left\{a_{n}\right\}_{n \geq 1}$ with terms in $K$. Pointwise sum and product give $S$ the structure of a commutative $K$-algebra with unit. We denote by $E: S \rightarrow S$ the $K$-algebra endomorphism $(E a)_{n}=a_{n+1}$ (the "shift operator").

For $\xi \in K^{*}$ denote by $e(\xi)$ the exponential sequence $e(\xi)_{n}=\xi^{n}$; note that $e(1)$ is the unit element of $S$. We will make use of the fact that for distinct $\xi_{1}, \ldots, \xi_{m}$ the sequences $e\left(\xi_{i}\right)$ are linearly independent over $K$ (the determinant $\left|e\left(\xi_{i}\right)_{j}\right|_{i, j=1}^{m}$ is Vandermonde). Denote by $\delta$ the sequence $\delta_{n}=n$. Then a sequence of the form (1.2) can be expressed

$$
a=\sum_{i=1}^{t} p_{i}(\delta) e\left(\mu_{i}\right)
$$

The proof of Theorem 1.5 will make use of the subalgebra

$$
R=K\left[a, E a, E^{2} a, \ldots\right] \subset S
$$

generated by the sequence $a$ together with its leftward shifts. This is a finitely generated $K$-algebra because $a$ obeys a linear recurrence. 
Let $Q$ be a commutative semigroup, written multiplicatively. The semigroup algebra $K[Q]$ has $K$-basis indexed by the elements of $Q$. The basis element corresponding to $\mu \in Q$ is written $[\mu]$. Multiplication is defined on basis elements by $[\mu][\lambda]=[\mu \lambda]$ and extended by linearity.

Lemma 2.1. Let $a_{n}$ be given by (1.2), and let $Q \subseteq K^{*}$ be the multiplicative semigroup generated by $\mu_{1}, \ldots, \mu_{t}$.

(1) There is an inclusion of $K$-algebras $R \hookrightarrow K[Q][x]$.

(2) If the sequence $a_{n}$ obeys a simple linear recurrence, there is an isomorphism of $K$-algebras $R \simeq K[Q]$.

Proof. Write $\ell=\sum\left(\operatorname{deg} p_{i}+1\right)$. Since $a$ obeys a linear recurrence of length $\ell+1$, we have

$$
R=K\left[a, E a, \ldots, E^{\ell-1} a\right] .
$$

Since $E e(\xi)=\xi e(\xi)$ and $E \delta=\delta+1$, by (2.1) we have

$$
E^{j} a=\sum_{i=1}^{t} p_{i}(\delta+j) \mu_{i}^{j} e\left(\mu_{i}\right),
$$

and hence there is an inclusion $R \subseteq R^{\prime}[\delta]$, where

$$
R^{\prime}=K\left[e\left(\mu_{1}\right), \ldots, e\left(\mu_{t}\right)\right] .
$$

Since $e\left(\mu_{i}\right) e\left(\mu_{j}\right)=e\left(\mu_{i} \mu_{j}\right)$ the linear map $e: K[Q] \rightarrow R^{\prime}$ sending $\left[\mu_{i}\right] \mapsto e\left(\mu_{i}\right)$ is a $K$-algebra homomorphism. Since the exponential sequences $e\left(\mu_{i}\right)$ are linearly independent, it is an isomorphism. Thus $R^{\prime} \simeq K[Q]$.

If $\left\{a_{n}\right\}$ satisfies a simple linear recurrence, then the polynomials $p_{i}(n)$ are nonzero constants. From (2.3) we have

$$
E^{j} a=\sum_{i=1}^{t} p_{i} \mu_{i}^{j} e\left(\mu_{i}\right)
$$

Thus the linear span of the sequences $a, E a, \ldots, E^{\ell-1} a$ coincides with that of $e\left(\mu_{1}\right)$, $\ldots, e\left(\mu_{\ell}\right)$ (the transition matrix is the product of a Vandermonde and an invertible diagonal matrix). It follows from (2.2) that

$$
R=K\left[e\left(\mu_{1}\right), \ldots, e\left(\mu_{t}\right)\right]=R^{\prime} \simeq K[Q] .
$$

This completes the proof of (2).

To prove (1), it suffices to show that $\delta$ is transcendental over $R^{\prime}$. Suppose it had algebraic degree $m$. From among the algebraic relations

$$
\rho_{0} \delta^{m}+\rho_{1} \delta^{m-1}+\cdots+\rho_{m}=0, \quad \rho_{j} \in R^{\prime}, \rho_{0} \neq 0,
$$

writing

$$
\rho_{0}=\sum_{i=1}^{s} b_{i} e\left(\xi_{i}\right), \quad b_{i} \in K^{*}, \xi_{i} \in Q,
$$

choose a relation in which the number of terms $s$ in the leading coefficient $\rho_{0}$ is minimal. This minimality forces $e\left(\xi_{1}\right), \ldots, e\left(\xi_{s}\right)$ to be linearly independent; equivalently, the $\xi_{i}$ must be distinct. Multiplying (2.6) by $b_{s}^{-1} e\left(\xi_{s}^{-1}\right)$ we may assume that $b_{s}=\xi_{s}=1$. 
Suppose first that $s>1$. Applying the difference operator $\Delta=E-1$ to (2.6) we obtain

$$
E\left(\rho_{0}\right)(\delta+1)^{m}-\rho_{0} \delta^{m}+q(\delta)=0,
$$

where $q \in R^{\prime}[x]$ is a polynomial of degree at most $m-1$. Thus

$$
\begin{aligned}
0 & =\sum_{i=1}^{s} b_{i} \xi_{i} e\left(\xi_{i}\right)(\delta+1)^{m}-\sum_{i=1}^{s} b_{i} e\left(\xi_{i}\right) \delta^{m}+q(\delta) \\
& =\sum_{i=1}^{s-1} b_{i}\left(\xi_{i}-1\right) e\left(\xi_{i}\right) \delta^{m}+q(\delta)+\tilde{q}(\delta)
\end{aligned}
$$

in which $\tilde{q} \in R^{\prime}[x]$ again has degree at most $m-1$. By the minimality of $s$, the coefficient of $\delta^{m}$ must vanish, and this contradicts the linear independence of the sequences $e\left(\xi_{i}\right)$.

It remains to consider the case $s=1$. By our rescaling convention, $b_{1}=\xi_{1}=1$, hence $\rho_{0}=e(1)$. Writing $\rho_{1}=\sum_{i=1}^{s^{\prime}} c_{i} e\left(\psi_{i}\right)$ for distinct $\psi_{i}$, applying $\Delta$ to the relation (2.6), we obtain

$$
e(1)\left[(\delta+1)^{m}-\delta^{m}\right]+\sum_{i=1}^{s^{\prime}} c_{i}\left[\psi_{i} e\left(\psi_{i}\right)(\delta+1)^{m-1}-e\left(\psi_{i}\right) \delta^{m-1}\right]+p(\delta)=0
$$

where $p \in R^{\prime}[x]$ is a polynomial of degree at most $m-2$. The coefficient of $\delta^{m-1}$ is

$$
m e(1)+\sum_{i=1}^{s^{\prime}}\left(\psi_{i}-1\right) e\left(\psi_{i}\right)=0
$$

If $s^{\prime}=0$ we obtain $m e(1)=0$, a contradiction as $K$ has characteristic 0 ; if $s^{\prime}=1$ and $\psi_{1}=1$ we obtain the same contradiction. Finally, if some $\psi_{i} \neq 1$, then (2.7) contradicts the linear independence of the sequences $e\left(\psi_{i}\right)$ and $e(1)$.

Lemma 2.2. Let $A$ be a finitely generated abelian group, and fix a set of generators $q_{1}, \ldots, q_{\ell}$ for $A$. Let $Q \subset A$ be the semigroup generated by the $q_{i}$. The following are equal:

(1) The rank of $A$.

(2) The Krull dimension of $K[Q]$.

(3) The maximum number of elements in $K[Q]$ algebraically independent over $K$.

Proof. Write $A=B \oplus C$, with $B$ finite and $C$ free abelian. By Maschke's Theorem, $K[B]$ is a finite product of copies of $K$, hence $K[A] \simeq K[B] \otimes_{K} K[C]$ is a finite product of copies of $K[C]$. Since $K[C]$ is a Laurent polynomial ring, it follows that $K[A]$ is reduced, and hence $K[Q]$ is reduced. Now by [2, p. 466], the maximum number of algebraically independent elements in $K[Q]$ is equal to its Krull dimension. Finally, by Proposition 7.5 in [12], the Krull dimension of $K[Q]$ is equal to the rank of $A$.

Proof of Theorem 1.5. By Lemma 2.1, we have $R \hookrightarrow K[Q][x] \simeq K[Q \times \mathbb{N}]$. Thus by Lemma 2.2, the maximum number of algebraically independent elements in $R$ is at $\operatorname{most} \operatorname{rank}(A \times \mathbb{Z})=r+1$. In particular, the $r+2$ elements $a, E a, \ldots, E^{r+1} a \in R$ are algebraically dependent over $K$; that is, the sequence $a$ obeys a polynomial recurrence of length $r+2$. 
If $a$ satisfies a simple linear recurrence, then $R \simeq K[Q]$ by Lemma 2.1 and the elements $a, E a, \ldots, E^{r} a$ are algebraically dependent over $K$, so $a$ obeys a polynomial recurrence of length $r+1$.

We now turn to the proof of Theorem 1.8. The key step uses Dodgson's rule [15] relating the determinant of an $\ell \times \ell$ matrix to its four corner $\ell-1 \times \ell-1$ minors and its central $\ell-2 \times \ell-2$ minor. For Toeplitz matrices the rule assumes a particularly simple form: with $A_{\ell, m}$ defined as in (1.3), we have for $2 \leq \ell \leq m$

$$
\operatorname{det} A_{\ell, m} \operatorname{det} A_{\ell-2, m}=\left(\operatorname{det} A_{\ell-1, m}\right)^{2}-\operatorname{det} A_{\ell-1, m-1} \operatorname{det} A_{\ell-1, m+1} \text {. }
$$

Proof of Theorem 1.8. We induct on $\ell$. If $x_{1} a_{n}+x_{2} a_{n+1}+\cdots+x_{\ell} a_{n+\ell-1}=0$ for all $n \geq 1$, then the vector $\left[x_{1}, \ldots, x_{\ell}\right]^{T}$ lies in the kernel of every $A_{\ell, n}$. Conversely, suppose $A_{\ell, n}$ is singular for all $n \geq \ell$. For each such $n$, let $\mathbf{x}_{n}=\left[x_{n 1}, \ldots, x_{n \ell}\right]^{T}$ be a nonzero vector in the kernel of $A_{\ell, n}$, and consider the $2 \times \ell$ matrices

$$
X_{n}=\left[\begin{array}{c}
\mathbf{x}_{n}^{T} \\
\mathbf{x}_{n+1}^{T}
\end{array}\right]=\left[\begin{array}{ccc}
x_{n 1} & \cdots & x_{n \ell} \\
x_{n+1,1} & \cdots & x_{n+1, \ell}
\end{array}\right] .
$$

If every $X_{n}$ has rank one, then each vector $\mathbf{x}_{n}$ is a scalar multiple of $\mathbf{x}_{\ell}$, and $\left\{a_{n}\right\}$ satisfies the linear recurrence $x_{\ell 1} a_{n}+\cdots+x_{\ell \ell} a_{n+\ell-1}=0$.

Suppose now that some $X_{n}$ has rank two. The transposes of the first $\ell-1$ row vectors of $A_{\ell, n}$ all lie in the kernel of $X_{n}$, so they must be linearly dependent. In particular, the upper-left minor $\operatorname{det} A_{\ell-1, n}$ vanishes. We now induct forwards and backwards on $m$ to show that $\operatorname{det} A_{\ell-1, m}$ vanishes for all $m \geq \ell$. The lefthand side of (2.8) is zero since $A_{\ell, m}$ is singular. Therefore, if either $\operatorname{det} A_{\ell-1, m-1}$ or $\operatorname{det} A_{\ell-1, m+1}$ vanishes, then $\operatorname{det} A_{\ell-1, m}$ must vanish as well. This completes the induction on $m$. By induction on $\ell$, the sequence $\left\{a_{n}\right\}_{n \geq 2}$ satisfies a linear recurrence of length at most $\ell-1$. This trivially implies that $\left\{a_{n}\right\}_{n \geq 1}$ satisfies a linear recurrence of length at most $\ell$.

\section{Determinant FACTORIZATIONS}

If $\left\{a_{n}\right\}_{n \geq 1}$ satisfies a simple linear recurrence of length $\ell+1$, the determinants $\operatorname{det} A_{\ell, n}$ have a simple closed form. Note that $\ell+1$ is the minimum length for which these determinants do not vanish, by Theorem 1.8 .

Theorem 3.1. Let $\mu_{i}, c_{i} \in K^{*}(i=1, \ldots, \ell)$, and set $a_{n}=\sum_{i=1}^{\ell} c_{i} \mu_{i}^{n}$. The determinant of the $\ell \times \ell$ Toeplitz matrix $A_{\ell, n}$ defined in (1.3) has the factorization

$$
\operatorname{det} A_{\ell, n}=(-1)^{\ell(\ell-1) / 2} c_{1} \cdots c_{\ell}\left(\mu_{1} \cdots \mu_{\ell}\right)^{n-\ell+1} \prod_{i<j}\left(\mu_{i}-\mu_{j}\right)^{2} .
$$

Proof. Consider the Vandermonde matrices $V=\left(\mu_{i}^{j-1}\right)_{i, j=1}^{\ell}$ and $V^{\prime}=\left(\mu_{j}^{\ell-i}\right)_{i, j=1}^{\ell}$, and let $D$ be the diagonal matrix with diagonal entries $c_{i} \mu_{i}^{n-\ell+1}, i=1, \ldots, \ell$. The $(i, j)$-entry of the product $V^{\prime} D V$ is then

$$
\left(V^{\prime} D V\right)_{i, j}=\sum_{k=1}^{\ell} \mu_{k}^{\ell-i} c_{k} \mu_{k}^{n-\ell+1} \mu_{k}^{j-1}=\sum_{k} c_{k} \mu_{k}^{n-i+j}=a_{n-i+j} .
$$

Thus $A_{\ell, n}=V^{\prime} D V$. Since $V^{\prime}$ differs from $V^{T}$ by a row permutation of length $\ell(\ell-1) / 2$ we obtain

$$
\operatorname{det} A_{\ell, n}=(-1)^{\ell(\ell-1) / 2} \operatorname{det} D(\operatorname{det} V)^{2},
$$


which yields (3.1).

It seems likely that Theorem 3.1 can be extended to cover the situation of an arbitrary linear recurrence. One difficulty is that in general, the factors $\mu_{i}-\mu_{j}$ occur with multiplicity. For example, if

$$
a_{n}=\left(b n^{3}+c n^{2}+d n+e\right) \mu_{1}^{n}+(f n+g) \mu_{2}^{n}+h \mu_{3}^{n},
$$

then

$$
\operatorname{det} A_{7, n}=1296 b^{4} f^{2} h \mu_{1}^{4 n-12} \mu_{2}^{2 n-8} \mu_{3}^{n-6}\left(\mu_{1}-\mu_{2}\right)^{16}\left(\mu_{1}-\mu_{3}\right)^{8}\left(\mu_{2}-\mu_{3}\right)^{4} .
$$

Of particular interest is the special case when $a_{n}$ is a polynomial function of $n$.

Proposition 3.2. Let $p(x)$ be a monic polynomial of degree $\ell$. As a polynomial in $x$, the $(\ell+1) \times(\ell+1)$ Toeplitz determinant $\Delta(x)=\operatorname{det}(p(x-i+j))_{i, j=0}^{\ell}$ equals the constant $\ell !^{\ell+1}$, independent of $p$.

The proof of this proposition will make use of the following generalization of the Vandermonde determinant 11.

Lemma 3.3. Let $p_{j}(x)$ be polynomials $(j=0, \ldots, \ell)$ with leading coefficients $a_{j}$ and $\operatorname{deg}\left(p_{j}\right)=\ell-j$. If $y_{0}, \ldots, y_{\ell}$ are indeterminates, then

$$
\operatorname{det}\left(p_{j}\left(y_{i}\right)\right)_{i, j=0}^{\ell}=a_{0} \cdots a_{\ell} \prod_{i<j}\left(y_{i}-y_{j}\right) .
$$

Proof. Perform elementary column operations from right to left, reducing the determinantal calculation to that of the Vandermonde identity.

Proof of Proposition 3.2. Set $q_{1}(x)=\frac{p(x+1)-p(x)}{\ell}$, which is monic of degree $\ell-1$. Subtracting the first column from the second, the second from the third, and so on, we obtain

$$
\Delta(x)=\ell^{\ell}\left|\begin{array}{cccc}
p(x) & q_{1}(x) & \cdots & q_{1}(x+\ell-1) \\
p(x-1) & q_{1}(x-1) & \cdots & q_{1}(x+\ell-2) \\
\vdots & \vdots & \ddots & \vdots \\
p(x-\ell) & q_{1}(x-\ell) & \cdots & q_{1}(x-1)
\end{array}\right| .
$$

Next, set $q_{i+1}(x)=\frac{q_{i}(x+1)-q_{i}(x)}{\ell-i}$ for $i=1, \ldots, \ell-1$ and repeat the above reductions, initiating the column operations at the $(i+1)$-st column. At the conclusion of this process, we end up with the determinant

$$
\Delta(x)=\ell^{\ell}(\ell-1)^{\ell-1} \cdots 1^{1}\left|\begin{array}{cccc}
p(x) & q_{1}(x) & \cdots & q_{\ell}(x) \\
p(x-1) & q_{1}(x-1) & \cdots & q_{\ell}(x-1) \\
\vdots & \vdots & \ddots & \vdots \\
p(x-\ell) & q_{1}(x-\ell) & \cdots & q_{\ell}(x-\ell)
\end{array}\right|
$$

Finally, setting $y_{i}=x-i$ in the statement of Lemma 3.3, it follows that

$$
\Delta(x)=\prod_{k=1}^{\ell} k^{k} \cdot \prod_{0 \leq i<j \leq \ell}(j-i)=\prod_{k=1}^{\ell} k^{k} \cdot \prod_{k=1}^{\ell} k^{\ell+1-k}=\ell !^{\ell+1} .
$$

Applying Theorem 3.1 to the cyclic resultants $r_{n}$ given in (1.1) we obtain the following. 
Proposition 3.4. The determinant of the $2^{d} \times 2^{d}$ Toeplitz matrix $R_{n}=\left(r_{n-i+j}\right)_{i, j=1}^{2^{d}}$ has the factorization

$$
\operatorname{det} R_{n}=\left(\lambda_{1} \cdots \lambda_{d}\right)^{2^{d-1}\left(n-2^{d-1}\right)} \prod_{S, T}\left(\lambda_{S}-\lambda_{T}\right)^{2^{d-|S \cup T|}},
$$

where $\lambda_{S}:=\prod_{i \in S} \lambda_{i}$, and the product is taken over all ordered pairs of disjoint subsets $S, T \subseteq\{1, \ldots, d\}$, not both empty.

Proof. Expanding the product (1.1) yields $r_{n}=\sum_{S \subseteq\{1, \ldots, d\}}(-1)^{d-|S|} \lambda_{S}^{n}$. Since $\prod_{S} \lambda_{S}=\left(\lambda_{1} \cdots \lambda_{d}\right)^{2^{d-1}}$, by Theorem 3.1 with $\ell=2^{d}$ we have

$$
\operatorname{det} R_{n}=\left(\lambda_{1} \cdots \lambda_{d}\right)^{2^{d-1}\left(n-2^{d}+1\right)} \prod_{S \neq T}\left(\lambda_{S}-\lambda_{T}\right) .
$$

For each pair of subsets $S, T \subseteq\{1, \ldots, d\}$ write $S^{\prime}=S-S \cap T, T^{\prime}=T-S \cap T$. Then $\lambda_{S}-\lambda_{T}=\lambda_{S \cap T}\left(\lambda_{S^{\prime}}-\lambda_{T^{\prime}}\right)$. Each ordered pair $\left(S^{\prime}, T^{\prime}\right)$ of disjoint subsets of $\{1, \ldots, d\}$, not both empty, arises in this way from $2^{d-\left|S^{\prime} \cup T^{\prime}\right|}$ different ordered pairs $(S, T), S \neq T$. The product in (3.3) can therefore be rewritten as

$$
\prod_{S \neq T}\left(\lambda_{S}-\lambda_{T}\right)=\prod_{S \neq T} \lambda_{S \cap T} \prod_{\substack{\left.S^{\prime} \cap T^{\prime}=\emptyset \\ S^{\prime}, T^{\prime}\right) \neq(\emptyset, \emptyset)}}\left(\lambda_{S^{\prime}}-\lambda_{T^{\prime}}\right)^{2^{d-\left|S^{\prime} \cup T^{\prime}\right|}} .
$$

Every element $i \in\{1, \ldots, d\}$ is contained in $4^{d-1}-2^{d-1}$ intersections of the form $S \cap T, S \neq T$. Thus, the exponent on $\lambda_{1} \cdots \lambda_{d}$ is $2^{d-1}\left(n-2^{d}+1\right)+4^{d-1}-2^{d-1}=$ $2^{d-1}\left(n-2^{d-1}\right)$.

Remark 3.5. The degree of $\operatorname{det} R_{n}$ as a polynomial in the roots $\lambda_{1}, \ldots, \lambda_{d}$ is significantly smaller than might be expected. Every term in the expansion of $R_{2^{d}}$ has degree $d 4^{d}$. On the other hand, by (3.3) the degree of $\operatorname{det} R_{2^{d}}$ is only $M(d):=$ $\sum_{S, T \subseteq\{1, \ldots, d\}} \max \{|S|,|T|\}$. It is easily seen (e.g. by the weak law of large numbers) that $M(d) \sim \frac{1}{2} d 4^{d}$ as $d \rightarrow \infty$. For large $d$, so much cancellation occurs in the expansion of $\operatorname{det} R_{2^{d}}$ that its degree is approximately cut in half.

\section{Cyclic Resultants}

We are now in a position to prove the first two results stated in the Introduction. Expanding the product formula (1.1) yields

$$
r_{n}=\sum_{S \subset\{1, \ldots, d\}}(-1)^{d-|S|} \lambda_{S}^{n}
$$

where $\lambda_{S}=\prod_{i \in S} \lambda_{i}$. Thus the sequence $r_{n}$ obeys a simple linear recurrence of length $2^{d}$. Note, however, that the coefficients are functions of the $\lambda_{i}$.

If $f$ is reciprocal of even degree $d$, its roots come in reciprocal pairs $\lambda_{1}^{ \pm 1}, \ldots, \lambda_{d / 2}^{ \pm 1}$, and the expansion of (1.1) yields

$$
r_{n}=\sum_{S, T} 2^{\frac{d}{2}-|S \cup T|}(-1)^{|S \cup T|}\left(\frac{\lambda_{S}}{\lambda_{T}}\right)^{n},
$$

where the sum is over disjoint subsets of $S, T \subset\{1, \ldots, d / 2\}$. 
Proof of Theorem 1.1. We take "generic" to mean that $f$ does not have a root of unity as a zero and that no two products of distinct subsets of the roots of $f$ are equal; that is, $\lambda_{S} \neq \lambda_{T}$ for distinct subsets $S, T \subset\{1, \ldots, d\}$. The first author, in Corollary 1.7 of [8], extending work of Fried [6], proves that a generic monic polynomial $f(x) \in K[x]$ is determined by its full sequence $\left\{r_{n}\right\}_{n \geq 1}$ of cyclic resultants. By (4.1) and Theorem 1.8, the sequence $\left\{r_{n}\right\}$ obeys a polynomial recurrence of length $2^{d+1}+1$ given by the vanishing of $\left(2^{d}+1\right) \times\left(2^{d}+1\right)$ Toeplitz determinants. Unlike the linear recurrence of length $2^{d}+1$, this recurrence is independent of the polynomial $f$. By minor expansion along the bottom row, the recurrence may be expressed in the form

$$
\left(\operatorname{det} R_{n}\right) \cdot r_{n+2^{d}+1}=P\left(r_{n-2^{d}+1}, \ldots, r_{n+2^{d}}\right), \quad n \geq 2^{d},
$$

for some polynomial $P \in K\left[x_{1}, \ldots, x_{2^{d+1}}\right]$, where $R_{n}=\left(r_{n+i-j}\right)_{i, j=1}^{2^{d}}$.

By Proposition [3.4, a generic polynomial $f$ gives rise to nonsingular $R_{n}$ for all $n$. Moreover, a straightforward calculation using (3.2) reveals that

$$
\operatorname{det} R_{n}=\frac{\left(\operatorname{det} R_{2^{d}+1}\right)^{n-2^{d}}}{\left(\operatorname{det} R_{2^{d}}\right)^{n-2^{d}-1}} .
$$

It follows from this and (4.2) that for $m \geq 2^{d}+2^{d}+1=2^{d+1}+1$, the resultant $r_{m}$ is determined by the resultants $r_{i}$ with $i<m$. In particular, the values $r_{1}, \ldots, r_{2^{d+1}}$ determine the full sequence of resultants, and hence they determine $f$.

If $f$ is reciprocal of even degree $d$, we take "generic" to mean that $f$ does not have a root of unity as a zero and that no two quotients of the form $\lambda_{S} / \lambda_{T}$ are equal, where $S$ and $T$ are disjoint subsets of $\{1, \ldots, d / 2\}$. As there are $3^{d / 2}$ such pairs $(S, T)$, by Theorem 1.8 , the sequence $\left\{r_{n}\right\}$ obeys a polynomial recurrence of length $2 \cdot 3^{d / 2}+1$ given by the vanishing of $\left(3^{d / 2}+1\right) \times\left(3^{d / 2}+1\right)$ Toeplitz determinants. Moreover, the matrices $R_{n}^{\prime}=\left(r_{n-i+j}\right)_{i, j=1}^{3^{d / 2}}$ are nonsingular by Theorem 3.1. In [8, Corollary 1.12], it is shown that the sequences of nonzero cyclic resultants generated by a reciprocal polynomial of degree $d$ determines it. The proof now proceeds by minor expansion as before.

Proof of Theorem 1.4. The elements $\lambda_{S} \in K$ appearing in (4.1) lie in the multiplicative subgroup $A \subseteq K^{*}$ generated by $\lambda_{1}, \ldots, \lambda_{d}$. By Theorem 1.5 applied to (4.1), the sequence $\left\{r_{n}\right\}$ obeys a polynomial recurrence of length rank $A+1 \leq d+1$.

If $f(x)$ is reciprocal, its roots $\lambda_{i}$ come in reciprocal pairs, and the rank of $A$ is at most $d / 2$. By Theorem [1.5 it follows that $\left\{r_{n}\right\}$ obeys a polynomial recurrence of length $d / 2+1$.

We close this section with an example illustrating the techniques used in proving Theorem 1.1. By (4.1) the resultants $r_{m}$ of a monic quadratic polynomial $f(x)=$ $x^{2}+a x+b$ satisfy a linear recurrence of length 5 . By Theorem 1.8 it follows that

$$
\operatorname{det} A_{5, n}=\left|\begin{array}{ccccc}
r_{n} & r_{n-1} & r_{n-2} & r_{n-3} & r_{n-4} \\
r_{n+1} & r_{n} & r_{n-1} & r_{n-2} & r_{n-3} \\
r_{n+2} & r_{n+1} & r_{n} & r_{n-1} & r_{n-2} \\
r_{n+3} & r_{n+2} & r_{n+1} & r_{n} & r_{n-1} \\
r_{n+4} & r_{n+3} & r_{n+2} & r_{n+1} & r_{n}
\end{array}\right|=0 .
$$


Minor expansion along the bottom row gives our polynomial recurrence

$$
r_{n+4}\left|\begin{array}{cccc}
r_{n-1} & r_{n-2} & r_{n-3} & r_{n-4} \\
r_{n} & r_{n-1} & r_{n-2} & r_{n-3} \\
r_{n+1} & r_{n} & r_{n-1} & r_{n-2} \\
r_{n+2} & r_{n+1} & r_{n} & r_{n-1}
\end{array}\right|=P\left(r_{n-4}, r_{n-3}, \ldots, r_{n+3}\right)
$$

expressing $r_{n+4}$ as a rational function in $r_{n-4}, \ldots, r_{n+3}$. For generic $f$, the determinant on the left side of (4.3) never vanishes, so the entire sequence of resultants is determined by the values of $r_{1}, \ldots, r_{8}$.

While (4.3) gives a polynomial recurrence for $r_{m}$ of length 9 , there in fact exists a recurrence of length 3 by Theorem 1.4. Explicitly, this recurrence is given by (5) below. The coefficients of this shorter recurrence depend on the polynomial $f$, whereas (4.3) gives a universal recurrence independent of $f$.

\section{Computations}

Here we list here explicit polynomial recurrences witnessing Theorem 1.4 in the cases $d=1$ and $d=2$. The cyclic resultants of a monic linear polynomial $f(x)=$ $x+a$ obey the length- 2 recurrence

$$
r_{n+1}=-a r_{n}-a-1 .
$$

For a monic quadratic $f(x)=x^{2}+a x+b$ we have the length-3 polynomial recurrence

$$
\begin{aligned}
&(a+b+1)\left[(a-2) r_{n+2}+a(a-b-1) r_{n+1}+(a-2 b) b r_{n}-(a-b-1)(a+b+1)\right] \\
&=-r_{n+2}^{2}-(a-2 b) r_{n+1} r_{n+2}-a b r_{n} r_{n+2}+(a-b-1) b r_{n+1}^{2} \\
& \quad-(a-2) b^{2} r_{n} r_{n+1}-b^{3} r_{n}^{2} .
\end{aligned}
$$

We close with the explicit reconstructions of polynomials from their cyclic resultants in the cases $d=2,3$. For quadratic $f=x^{2}+a x+b$, two nonzero resultants suffice to determine $a, b$ :

$$
a=\frac{r_{1}^{2}-r_{2}}{2 r_{1}}, b=\frac{r_{1}^{2}-2 r_{1}+r_{2}}{2 r_{1}} .
$$

For cubic $f=x^{3}+a x^{2}+b x+c$, four nonzero resultants give inversion:

$$
\begin{aligned}
& a=\frac{-12 r_{2} r_{1}^{3}-12 r_{1} r_{2}^{2}+3 r_{2}^{3}-r_{2} r_{1}^{4}-8 r_{2} r_{1} r_{3}+6 r_{1}^{2} r_{4}}{24 r_{2} r_{1}^{2}}, b=\frac{-r_{1}^{2}-2 r_{1}+r_{2}}{2 r_{1}}, \\
& c=\frac{-3 r_{2}^{3}+r_{2} r_{1}^{4}+8 r_{2} r_{1} r_{3}-6 r_{1}^{2} r_{4}}{24 r_{1}^{2} r_{2}} .
\end{aligned}
$$

In addition, a monic quartic polynomial can be explicitly reconstructed using five resultants; however, the expressions are too cumbersome to list here. We remark that the pattern of monomial denominators found in the inversions above does not continue for higher degree reconstructions.

\section{REFERENCES}

[1] J. H. Conway and R. Guy, The Book of Numbers, Springer-Verlag, 1996. MR1411676 (98g:00004)

[2] D. Cox, J. Little, and D. O'Shea, Ideals, varieties, and algorithms, second ed., Undergraduate Texts in Mathematics, Springer-Verlag, New York, 1997. MR.1417938 (97h:13024)

[3] J.J. Duistermaat and V. Guillemin, The spectrum of positive elliptic operators and periodic bicharacteristics, Inv. Math. 25 (1975) 39-79. MR0405514(53:9307) 
[4] S. Elaydi, An Introduction to Difference Equations, Springer, 1999. MR:1711587 (2001g:39001)

[5] G. Everest and T. Ward. Heights of Polynomials and Entropy in Algebraic Dynamics. Springer-Verlag London, Ltd., London, 1999. MR.1700272 (2000e:11087)

[6] D. Fried, Cyclic resultants of reciprocal polynomials, in Holomorphic Dynamics (Mexico 1986), Lecture Notes in Math. 1345, Springer-Verlag, 1988, 124-128. MR0980956|(90h:57004)

[7] V. Guillemin, Wave trace invariants, Duke Math. J. 83 (1996), 287-352. MR.1390650 (97f:58131)

[8] C. Hillar, Cyclic resultants, J. Symb. Comp. 39 (2005), 653-669; erratum 40 (2005), 11261127. MR 2167674

[9] A. Iantchenko, J. Sjöstrand, and M. Zworski, Birkhoff normal forms in semi-classical inverse problems, Math. Res. Lett. 9 (2002), 337-362. MR1909649(2003f:35284)

[10] K. Kedlaya, Computational Complexity 15 (2006), 1-19. MR2226067

[11] C. Krattenthaler, "Advanced Determinant Calculus," Sem. Lothar. Combin. 42 (1999), Art. B42q. MR1701596 (2002i:05013)

[12] E. Miller and B. Sturmfels, Combinatorial Commutative Algebra, Springer, 2004. MR2110098 (2006d:13001)

[13] K. Purbhoo, A Nullstellensatz For Amoebas, preprint.

[14] W. H. Stevens, Recursion formulas for some abelian knot invariants, Journal of Knot Theory and Its Ramifications, Vol. 9, No. 3 (2000) 413-422.

[15] D. Zeilberger, Dodgson's determinant-evaluation rule proved by two-timing men and women, Elec. J. Comb. 4(2), 1997.

Department of Mathematics, Texas A \& M University, College Station, TX 77843

E-mail address: chillar@math.tamu.edu

Department of Mathematics, University of California, Berkeley, California 94720

E-mail address: levine@math.berkeley.edu 